\title{
Antecedents of Loyalty in Mobile Social Network Games

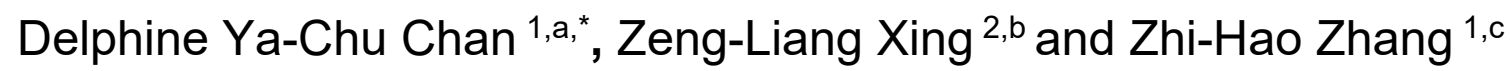 \\ ${ }^{1}$ School of Business, Beijing Institute of Technology, Zhuhai \\ ${ }^{2}$ Beijing Foreign Enterprise Human Resources Service Co., Ltd. \\ aw-chu119@qq.com, bzliang.xing@partner.samsung.com, c616689094@qq.com \\ ${ }^{*}$ Corresponding author
}

Keywords: mSNGs; Loyalty; Trust; Information; Interaction; Playfulness

\begin{abstract}
In view of the economic potential and continuous innovation of mobile social network games (mSNGs), this paper uses the questionnaire survey to collect 389 valid samples from the "Honour of kings" game, which is ranked first in global mobile game revenue in 2017, to explore the antecedents of loyalty in mobile game. The results show that the information, interaction and playfulness of sSNGs have significantly positive impacts on the trust and loyalty of the players. Among them, the playfulness has the most significantly direct effect on trust and loyalty, and the trust has the partially mediating effect. The results are helpful for the product design and continuous development of mSNGs. It is also proved that the playfulness and trust are the important influencing factors of loyalty.
\end{abstract}

\author{
手游忠诚度的前因研究 \\ 詹雅竹 ${ }^{1, a,{ }^{*}}$, 邢增亮 ${ }^{2, b}$, 张志豪 ${ }^{1, c}$ \\ 1 北京理工大学珠海学院商学院, 珠海, 广东, 中国 \\ 2北京外企市场营销顾问有限公司, 北京, 中国 \\ a w-chu119@qq.com, b zliang.xing@partner.samsung.com, c616689094@qq.com \\ *通讯作者
}

关键词：移动社交游戏; 忠诚度; 信任; 资讯性; 互动性; 娱乐性

中文摘要. 鉴于移动社交游戏的经济潜力与持续创新, 本文以 2017 年全球手游收入第一的 “王者荣耀” 为例, 采用问卷调查法搜集389份有效样本, 探讨影响手游忠诚度的前因。结果 显示, 手游的资讯性、互动性与娱乐性对玩家的信任和忠诚度皆有正向显著影响, 其中又以 娱乐性对信任及忠诚度的影响最显著, 而信任则具有部份中介效果。研究结果对mSNGs的 产品设计与持续发展有实质助益, 也验证了娱乐性和信任是影响其忠诚度的重要因素。

\section{1. 引言}

与过去相比，消费者花费在游戏的时间与金钱比以往更甚。游戏不仅是娱乐活动，其趣 味性在社交、教育、广告媒体、商务运营等方面也具有一定的效用 ${ }^{[1-3]}$ 。 mSNGs 是一种在社会 网络服务(social network services, SNS)运行下使用移动设备和亲友互动的社交游戏app, 通过 游戏玩家之间的信息传播、话题讨论与竞争协作可增强在现实生活中的关系和联系 ${ }^{[4]}$ 。随着 移动设备与游戏开发的增加, 网游产业逐渐由官方宣传的传统分销渠道转为用户口碑的社会 化传播渠道, 手游市场规模也大幅超越端游市场 ${ }^{[5-6]}$ 。因此, Facebook、Twitter、WeChat、QQ、 
等社交媒体成为人们与亲友之间传递信息、沟通交流、休闲娱乐和游戏竞技的重要平台， mSNGs在全球SNS发展中也日益普及 ${ }^{[4,7]}$ 。然而, 游戏的创新发展同时有益于教育学习 ${ }^{[8-11]}$ 和 营销传播 ${ }^{2,12-13]}$, 而且产品与技术不断地推陈出新, 因此从正面角度去探讨手游玩家忠诚度的 影响因素, 将有助于手游公司维系忠诚顾客以提升企业获利、降低争取新顾客的成本 ${ }^{[14]}$ 。

综上所述, 本文目的是通过mSNGs实证, 检验资讯性、互动性、娱乐性与信任对于玩家 忠诚度的影响效果, 为游戏厂商提供管理意涵, 以及增加忠诚度前因研究的理论意义。

\section{2. 文献回顾}

\section{1 资讯性、互动性和娱乐性}

资讯性(information)是为使用者提供丰富、具有帮助的信息, 而使用SNS的主要动机是从 相关渠道获得多种有用的信息, 包括及时从mSNGs取得促销服务、近期更新或其他所需的内 容 ${ }^{[7]}$ 。互动性(interaction)是指人、群体或事物之间的相互作用或影响 ${ }^{[15]}$ 。线上游戏的互动可 分为使用者一系统及使用者一使用者之间的互动, 人机互动能提供客制化、有意义的游戏体 验, 使用者之间的互动则是个体与他人进行沟通或社交活动的行为, 可由玩家参与社交活动 的频率来评估 ${ }^{[16]}$ 。娱乐性(playfulness)是个体在某段期间内的活动体验或对熟悉情景产生的乐 趣, 如同内在动机, 并随着时间、环境和互动状态的不同而有所变化。个体对网络环境若有 比较正面的兴趣, 表示其专注程度、愉悦感和好奇心比较强烈, 对网络互动也会更正面 ${ }^{[17-18]}$ 。

\section{2 信任和忠诚度}

信任(trust)是一种经济交换关系, 是长时间发展出来的直接经验 ${ }^{[19]}$ 。本研究将线上手游玩 家的信任定义为, 游戏玩家对于提供产品和服务的厂商, 经过长时间积累的信赖和信心程度。 忠诚度(loyalty) 是关于消费者透过对某一品牌认知、试用与重复等过程, 进而持续购买其产品 或服务程度 ${ }^{[20]}$; 无论线上或线下环境, 留住旧顾客比争取新顾客的成本还低, 而减少顾客流 失率亦可提高企业利润, 可见忠诚度的维系对于企业运营极为重要 ${ }^{[14,21]}$ 。

网络忠诚度(e-loyalty)是指消费者再次网购或重访网站的意向 ${ }^{[22-23]}$, 移动忠诚度(m-loyalty) 是指移动环境中的顾客忠诚 ${ }^{[22]}$ 。过去研究显示信息质量是移动网络中的关键因子, 并能强化 移动服务中的顾客忠诚 ${ }^{[24]}$; 互动性和娱乐性对使用态度有显著的正向影响 ${ }^{[18,25]}$, 甚至与信任、 移动忠诚度呈现高度相关 ${ }^{[16,22]}$ 。因此, 本文提出研究假设如下:

$\mathrm{H} 1$ ：资讯性、互动性和娱乐性对信任有正向影响

$\mathrm{H} 2$ : 资讯性、互动性和娱乐性对忠诚度有正向影响

H3：信任对忠诚度有正向影响。(a)资讯性会通过信任对忠诚度产生影响; (b)互动性会通 过信任对忠诚度产生影响; (c)娱乐性会通过信任对忠诚度产生影响。

\section{3. 研究方法}

“王者荣耀” 是2017年全球手游收入排名第一的 $\mathrm{mSNGs}^{[5]}$, 具有研究上的意义与代表性。 本文采用问卷调查法, 通过游戏的官方社群(WeChat, QQ, blog等)发放线上问卷, 确保受测者 都是 “王者荣耀” 的玩家。变量测题选自先前研究, 符合内容效度, 如表1所示; 资讯性、互 动性、娱乐性、信任与忠诚度等变量各有 5 题, 使用Likert 5 点量表。前测 50 个样本的信度分 析结果显示, 资讯性 $(\alpha=.949)$ 、互动性 $(\alpha=.970)$ 、娱乐性 $(\alpha=.954)$ 、信任 $(\alpha=.978)$ 和忠诚度 $(\alpha=.962)$ 皆具有高信度而适合发放正式问卷。正式问卷回收405份, 有效样本为389份。其中, 男性占 $55.53 \%$, 女性占 $44.47 \%$; 受测者多为 $21-30$ 岁 (64.52\%), 教育程度以专科和本科院校为主 $(68.38 \%)$, 月支配金额大多在 $1,001-3,000$ 元 $(49.87 \%)$, 日均游戏时数多为 $1-3$ 小时 $(57.84 \%)$ 。 


\section{4. 分析结果}

\section{1 信效度分析}

从表 1 可知, 所有变量的 Cronbach's $\alpha>0.7$, 项目总相关 $>0.5$, 因子载荷 $>0.7$, 组成信度 $>0.7$, AVE $>0.5$, 显示变量测题具有高度的内部一致性和良好的收玫效度。表 2 可见 AVE 开方后均 大于变量间的相关系数，显示变量间也有良好的区别效度。

表 1 信度与收敛效度

\begin{tabular}{|c|c|c|c|c|c|}
\hline \multirow[b]{2}{*}{ Construct \& Item } & \multicolumn{2}{|c|}{ Internal reliability } & \multicolumn{3}{|c|}{ Convergent validity } \\
\hline & Cronbach's $\alpha$ & $\begin{array}{c}\text { Item-total } \\
\text { correlation }\end{array}$ & $\begin{array}{c}\text { Factor } \\
\text { loading }\end{array}$ & $\begin{array}{l}\text { Composite } \\
\text { reliability }\end{array}$ & AVE \\
\hline \multicolumn{6}{|c|}{ Information $(\mathbf{M}=\mathbf{3 . 6 5 5} ; \mathbf{S D}=\mathbf{. 7 8 6})$ from Jeon et al. $(2016)^{[7]}$} \\
\hline INF1.提供完整的角色资讯 & .905 & .771 & .860 & .930 & .727 \\
\hline INF2.提供必备的活动资讯 & & .786 & .869 & & \\
\hline INF3.提供必须的改版资讯 & & .727 & .827 & & \\
\hline INF4.提供丰富的资讯内容 & & .788 & .869 & & \\
\hline INF5. 符合需求的资讯内容 & & .740 & .836 & & \\
\hline \multicolumn{6}{|c|}{ Interaction $(\mathbf{M}=\mathbf{3 . 6 8 6} ; \mathbf{S D}=\mathbf{. 8 1 0})$ from Choi and $\operatorname{Kim}(2004)^{[16]}$} \\
\hline INT1.能认识更多玩家 & .905 & .704 & .805 & .930 & .727 \\
\hline INT2.能和玩家们互动 & & .782 & .864 & & \\
\hline INT3.能增进玩家之间友谊 & & .809 & .885 & & \\
\hline INT4.能和队员产生协作 & & .774 & .863 & & \\
\hline INT5.能与队友分享经验 & & .747 & .844 & & \\
\hline \multicolumn{6}{|c|}{ Playfulness $(\mathbf{M}=\mathbf{3 . 6 9 6} ; \mathbf{S D}=\mathbf{. 7 6 3})$ from Moon and Kim $(2001)^{[18]}$} \\
\hline PLA1.引发游戏探索 & .890 & .741 & .842 & .920 & .697 \\
\hline PLA2.更新使我感兴趣 & & .751 & .849 & & \\
\hline PLA3.游戏模式有意思 & & .748 & .846 & & \\
\hline PLA4.游戏过程很享受 & & .756 & .848 & & \\
\hline PLA5.游戏过程释放压力 & & .672 & .786 & & \\
\hline \multicolumn{6}{|c|}{ Trust $(\mathbf{M}=3.476 ; \mathbf{S D}=.846)$ from Dwyer et al. $(1987)^{[19]}$} \\
\hline TRU1.值得相信的 & .929 & .815 & .885 & .946 & .779 \\
\hline TRU2.遵守承诺的 & & .857 & .913 & & \\
\hline TRU3. 有专业技能的 & & .794 & .870 & & \\
\hline TRU4.是高效的 & & .812 & .882 & & \\
\hline TRU5.是重视顾客的 & & .784 & .862 & & \\
\hline \multicolumn{6}{|c|}{ Loyalty $(\mathbf{M}=3.556 ; \mathbf{S D}=\mathbf{8 3 4})$ from Brown and Fassett $(1952)^{[20]}$ and Cyr et al. $(2006)^{[22]}$} \\
\hline 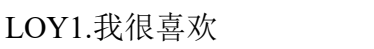 & .881 & .751 & .856 & .916 & .687 \\
\hline LOY2.我会反复玩 & & .767 & .870 & & \\
\hline LOY3.我会推荐朋友 & & .741 & .850 & & \\
\hline LOY4.不考虑他款手游 & & .631 & .747 & & \\
\hline LOY5.视为生活的一部分 & & .713 & .815 & & \\
\hline
\end{tabular}

\begin{tabular}{|c|c|c|c|c|c|}
\hline \multicolumn{6}{|c|}{ 表 2 区别效度 } \\
\hline Constructs & 1 & 2 & 3 & 4 & 5 \\
\hline 1. INF & .853 & & & & \\
\hline 2. INT & $.631 * *$ & .853 & & & \\
\hline 3. PLA & $.663 * *$ & $.712 * *$ & .835 & & \\
\hline 4. TRU & $.585 * *$ & $.616^{* *}$ & $.669 * *$ & .883 & \\
\hline 5. LOY & $.513 * *$ & $.531 * *$ & $.706 * *$ & $.599 * *$ & .829 \\
\hline
\end{tabular}

Note: The square roots of average variance extracted (AVEs) are shown in bold on the diagonal of the matrix; off diagonal elements are the correlations between constructs.

\section{2 假设检验}

回归分析结果显示，资讯性 $(\beta=.585, p<.001)$, 互动性 $(\beta=.616, p<.001)$ 与娱乐性 $(\beta=.669$, $p<.001$ ) 皆对信任有正向显著影响, 调整后整体模式解释力分别达到 $34 \%$ 、 $37.8 \%$ 和 $44.5 \%$, $\mathrm{F}$ 值分别为 201.095、138.334 和 384.597。即资讯性、互动性、娱乐性愈高, 玩家的信任愈高,

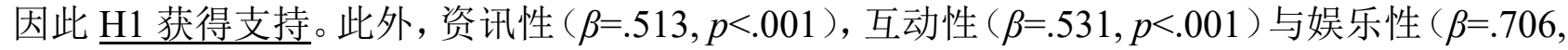
$p<.001$ ) 皆对忠诚度有正向显著影响, 调整后整体模式解释力分别达到 $26.1 \%$ 、 $28 \%$ 和 $34 \%$, 
$\mathrm{F}$ 值分别为 201.095、138.334 和 384.597。即资讯性、互动性、娱乐性愈高, 玩家的忠诚度愈 高, 故 $\mathrm{H} 2$ 也获得支持。

最后，信任对忠诚度有正向显著影响 $(\beta=.599, p<.001)$, 调整后整体模式解释为 $35.7 \%$, $\mathrm{F}$ 值为 216.580 , 表示信任愈高, 忠诚度也愈高。而在加入信任后, 资讯性 $(\beta=.248, p<.001)$, 互动性 $(\beta=.261, p<.001)$ 与娱乐性 $(\beta=.552, p<.001)$ 对忠诚度的影响仍显著，但系数值相较 于没加入信任时有所下降，而且调整后解释力分别为 $39.6 \% 、 39.8 \%$ 和 $52.5 \%, \mathrm{~F}$ 值分别为 128.204、129.255 和 215.573，VIF 值分别介于 $1.000 ~ 1.520 、 1.000 \sim 1.612$ 与 1.000 1.808, 没 有共线性的问题，因此可判定信任在资讯性、互动性与娱乐性对忠诚度的关系之间都具有部 分中介效果。即资讯性、互动性与娱乐性越高, 信任越高, 从而正向且部分影响忠诚度。故 $\underline{H} 3(a)(b)(c)$ 皆获得支持。

\section{5. 结论与管理意涵}

鉴于中国手游市场进入高速发展阶段并增速领先全球, 本研究以现象级手游 “王者荣耀” 的玩家为研究对象, 探讨其忠诚度与影响因素。结果显示, 玩家偏向年轻化, 男女比例接近, 而且手游的资讯性、互动性与娱乐性对于玩家的信任和忠诚度都有显著的影响; 尤其是玩家 的娱乐性愈高时, 其信任与忠诚度也愈强。娱乐性为手机游戏的基石, 在手游设计方面应辅 以大众化、互动性、丰富资讯性，并需保障玩家基本的游戏权益。未来研究则建议增加不同 手游类型的调查, 并扩大范围至其他国家或地区, 以大数据整合全球手游发展进程, 从而推 动相关研究的持续发展。

\section{致谢}

本文为广东省高校创新人才类项目（人文社科） 《基于可持续发展的组织营销与消费研 究》(2015WQNCX191)的阶段性成果之一。

\section{References}

[1] M. Kangas, Creative and playful learning: learning through game co-creation and games in a playful learning environment, Thinking Skills \& Creativity, vol.5, pp. 1-15, 2010.

[2] G. Killian and J. Hulland, Marketing promotions in social network games: making them work, Journal of Digital \& Social Media Marketing, vol.4, pp. 54-69, 2016.

[3] D. Spikol and M. Milrad, Physical activities and playful learning using mobile games, Research \& Practice in Technology Enhanced Learning, vol.3, pp. 275-295, 2008.

[4] D. H. Shin and Y. J. Shin, Why do people play social network games? Computers in Human Behavior, vol.27, pp. 852-861, 2011.

[5] iResearch, White paper of social distribution in Chinese online games, 2017.

[6] iResearch, China mobile e-sports report, 2017.

[7] H. Jeon, H. J. Ahn, and G. J. Yu, What makes people react to the posts on the brand pages of mobile social network games? Online Information Review, vol.40, pp. 435-448, 2016.

[8] Y. Baek and A Touati. Exploring how individual traits influence enjoyment in a mobile learning game, Computers in Human Behavior, vol.69, pp. 347-357, 2017.

[9] J. Huizenga, W. Admiraal, S. Akkerman, and G. T. Dam, Mobile game-based learning in secondary education: engagement, motivation and learning in a mobile city game, Journal of Computer Assisted Learning, vol.25, pp. 332-344, 2009. 
[10]M. Palomo-Duarte, A. Berns, A. Cejas, J. M. Dodero, J. A. Caballero and I Ruiz-Rube, Assessing foreign language learning through mobile game-based learning environments, Int. Journal of Human Capital \& Information Technology Professionals, vol.7, pp. 53-67, 2016.

[11] G. Schwabe and C. Göth, Mobile learning with a mobile game: design and motivational effects, Journal of Computer Assisted Learning, vol.21, pp. 204-216, 2005.

[12]R. Terlutter and M. L. Capella, The gamification of advertising: analysis and research directions of in-game advertising, advergames, and advertising in social network games, Journal of Advertising, vol.42, pp. 95-112, 2013.

[13]S. Verberckmoes, K. Poels, N. Dens, L. Herrewijn, and P. D. Pelsmacker, When and why is perceived congruity important for in-game advertising in fantasy games? Computers in Human Behavior, vol.64, pp. 871-880, 2016.

[14]F. F. Reichheld, W. E. Sasser, Zero defects: quality comes to services, Harvard Business Review, vol.68, pp. 105-111, 1990.

[15] Y. S. Su, W. L. Chiang, C. T. J. Lee, and H. C. Chang, The effect of flow experience on player loyalty in mobile game application, Computers in Human Behavior, vol.63, pp. 240-248, 2016.

[16]D. Choi and J. Kim, Why people continue to play online games: in search of critical design factors to increase customer loyalty to online contents, Cyberpsychology \& Behavior, vol.7, pp. 11-24, 2004.

[17]L. A. Barnett, The nature of playfulness in young adults, Personality \& Individual Differences, vol.4, pp. 949-958, 2007.

[18]J. W. Moon and Y. G. Kim, Extending the TAM for a world-wide-web context, Information \& Management, vol.38, pp. 217-230, 2001.

[19]F. R. Dwyer, P. H. Schurr, and S. Oh, Developing buyer-seller relationships, Journal of Marketing, vol.51, pp. 11-27, 1987.

[20]R. S. Brown and J. D. Fassett, Loyalty tests for admission to the bar, University of Chicago Law Review, vol.20, pp. 480-508, 1952.

[21]F. F. Reichheld and P. Schefter, E-loyalty: your secret weapon on the web, Harvard Business Review, vol.78, pp. 105-113, 2003.

[22]D. Cyr, M. Head, A. Ivanov, Design aesthetics leading to m-loyalty in mobile commerce, Information \& Management, vol.43, pp. 950-963, 2006.

[23]C. Flavián, M. Guinalíu, and R. Gurrea, The role played by perceived usability, satisfaction and consumer trust on website loyalty, Information \& Management, vol.43, pp. 1-14, 2006.

[24]M. Chae, J. Kim, H. Kim, and H. Ryu, Information quality for mobile internet services: a theoretical model with empirical validation, Electronic Markets, vol.12, pp. 38-46, 2002.

[25]A. Richmond, Enticing online shoppers to buy - a human behavior study, Computer Networks \& Isdn Systems, vol.28, pp. 1469-1480, 1996. 